\title{
SIMULATION OF THERMOMAGNETIC RECORDING PROCESS USING MAGNETIC FIELD MODULATION METHOD
}

\author{
T. Koyama, T. Kato, S. Iwata, and S. Tsunashima
}

Department of Electronics, Nagoya University, Furo-cho, Chikusa-ku, Nagoya 464-8603, Japan

Thermomagnetic recording process was simulated for $\mathrm{Tb}_{x}\left(\mathrm{Fe}_{78} \mathrm{Co}_{22}\right)_{100-x}$ media with the thickness of 40 and 20 $\mathrm{nm}$ by using Huth's model modified for the MFM recording. In the recording of marks smaller than $\sim 200 \mathrm{~nm}$, the recorded mark length was found to exhibit larger value than the designed mark. From the analysis of the effective fields acting on the domain wall, the stray field was found to be important factor in the recording of the small size marks.

Key words : thermomagnetic recording, magnetic field modulation, simulation, Huth's equation

\section{Introduction}

Magnetic amplifying magneto-optical system (MAMMOS) and domain wall displacement detection (DWDD) are considered to be the most promising candidates for next generation magneto-optical recording techniques [1,2]. In both techniques, small size marks (magnetic domains) are recorded by using magnetic field modulation (MFM), and marks as small as $\sim 100 \mathrm{~nm}$ are reported to be read out with high $\mathrm{S} / \mathrm{N}$ ratio. However, for further high density magneto-optical recording, it is very important to understand the thermomagnetic recording process during the formation of the marks smaller than $100 \mathrm{~nm}$ by MFM recording. In this study, we have simulated the thermomagnetic recording process by using Huth's model modified for the MFM recording.

\section{Simulation Method}

We assumed rectangular shaped domains separated by front and rear walls in the track direction ( $x$ direction) of the disk, but no side walls in the track-width direction ( $y$ direction). We also assumed one dimensional wall motion along $x$ direction. The temperature profile of the medium was calculated for TbFeCo films on PMMA substrate by assuming the three dimensional heat flow in the substrate [3]. For simplicity, when determining the stable wall position, the temperature was assumed to be uniform in the $y$ direction and in the film thickness direction as well. The laser spot size and linear velocity along the $x$ direction of the medium was assumed to be $1.0 \mu \mathrm{m}$ and $10 \mathrm{~m} / \mathrm{sec}$, respectively.

Stability condition of domain wall is given by the following equation [4]:

$$
\left|H_{\text {total }}=-\frac{1}{2 M_{s}} \frac{\partial \sigma_{w}}{\partial x}+H_{d}+H_{e x t}\right| \leq H_{c}
$$

where $x$ is the wall position, $M_{\mathrm{s}}$ the saturation magnetization, $H_{\mathrm{c}}$ the coercivity, $H_{\mathrm{d}}$ the demagnetization field, $H_{\mathrm{ext}}$ the external magnetic field, $\sigma_{\mathrm{w}}$ the energy density of domain wall. The $H_{\mathrm{d}}$ was the estimated value at the position of $y=0$, and was estimated by assuming that the magnetization in the region $|y| \leq 0.3$ contributes to the $H_{\mathrm{d}}$. The temperature dependence of $M_{\mathrm{s}}$ and $H_{\mathrm{c}}$ of $\mathrm{TbFeCo}$ for various $\mathrm{Tb}$ content are illustrated in Fig. 1 and Fig. 2, respectively. These profiles calculated from the magnetic parameters which obtained by the mean field fitting of the experimental results [5]. The temperature dependence of $\sigma_{\mathrm{w}}$ was approximately expressed

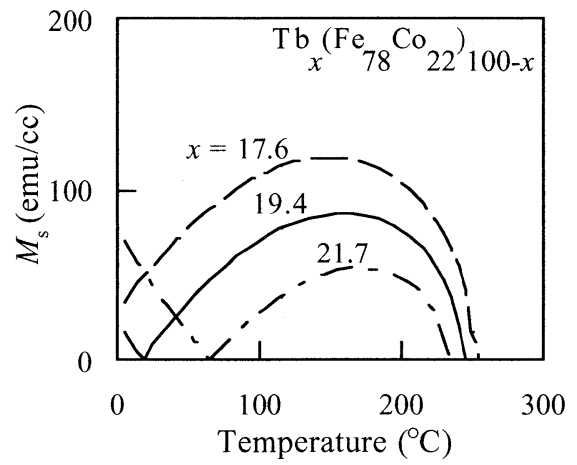

Fig. 1 Temperature dependence of saturation magnetization $M_{\mathrm{S}}$ for $\mathrm{Tb}_{x}\left(\mathrm{Fe}_{78} \mathrm{Co}_{22}\right)_{100-x}$.

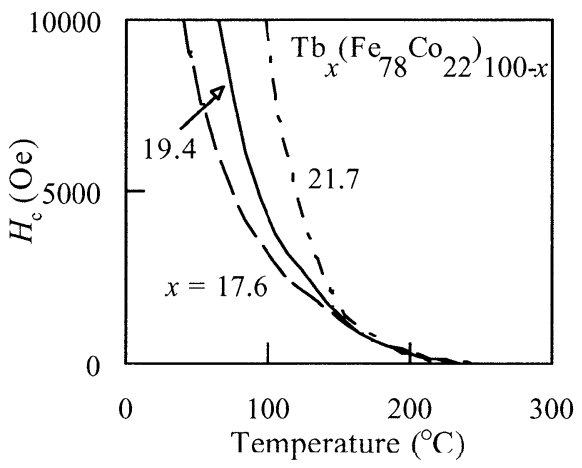

Fig. 2 Temperature dependence of coersivity $H_{\mathrm{c}}$ for $\mathrm{Tb}_{x}\left(\mathrm{Fe}_{78} \mathrm{Co}_{22}\right)_{100-x}$ 
by the following equation:

$$
\sigma_{w^{\prime}}(T)=2\left\{\left(T_{c}-T\right) /\left(T_{c}-T_{u m b}\right)\right\}^{1.6},
$$

where $T_{\mathrm{c}}$ is Curie temperature, $T$ the temperature of the media, and $T_{\mathrm{amb}}$ the ambient temperature.

\section{Results and Discussion}

Figure 3 shows recorded mark length vs designed mark length simulated for the formation of an isolated mark in the $\mathrm{Tb}_{x}\left(\mathrm{Fe}_{78} \mathrm{Co}_{22}\right)_{100-x}$ media with the thickness of $h=40 \mathrm{~nm}$, where the designed mark length means the ideal one corresponds to the wave form of the field modulation. The laser irradiation power and $H_{\text {ext }}$ are set to $3.0 \mathrm{~mW}$ and $\pm 200 \mathrm{Oe}$, respectively. The recorded mark length decreased with decreasing the designed mark length, but exhibited larger value than the designed mark. In the case of $x=19.4$, the recorded mark length was found to become $113 \mathrm{~nm}$ for the designed mark of $50 \mathrm{~nm}$, which is about two times as large as the designed value. This qualitatively agrees with the results of the domain observation for isolated recorded marks by using M-XTM [6]. The recorded mark length simulated for $x$ $=21.7$ became smaller compared to those of $x=17.6$ and 19.4. This compositional dependence of the recorded mark length is not clearly seen in the experimental observation [6], which may be due to the difference of the temperature profiles between in the experiment and in our simulation.

In order to understand the domain formation process, we have investigated the dependence of the effective fields acting on the magnetic wall. From the analysis of the effective fields corresponding to the terms in eq. (1), the stray field was found to be important factor in the recording of the small size marks. For writing smaller marks, larger stray fields which tend to expand the mark length are found. The stray field acting on the wall decreased with increasing $\mathrm{Tb}$ con

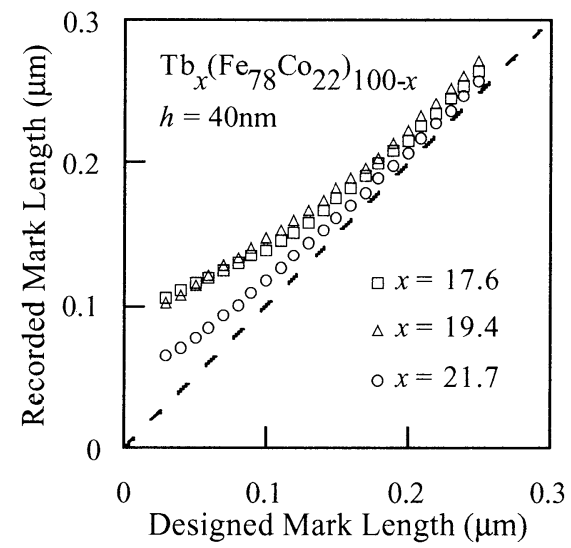

Fig. 3 Recorded mark length vs designed mark length simulated for the formation of an isolated mark in the $\mathrm{Tb}_{x}\left(\mathrm{Fe}_{78} \mathrm{Co}_{22}\right)_{100-x}$ medium with the thickness of $h=40 \mathrm{~nm}$.

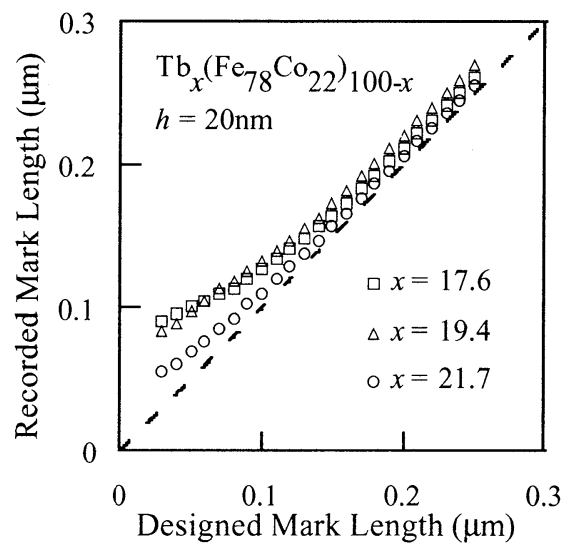

Fig. 4 Recorded mark length vs designed mark length simulated for the formation of an isolated mark in the $\mathrm{Tb}_{x}\left(\mathrm{Fe}_{78} \mathrm{Co}_{22}\right)_{100-x}$ medium with the thickness of $h=20 \mathrm{~nm}$.

tent $x$ because of the decrease of magnetization near the Curie point as shown in Fig. 1. The reason why the smallest mark length was recorded for $x=21.7$ can be understood by the decrease of the stray field acting on the domain wall.

The stray field is considered to decrease with decreasing the film thickness. In order to investigate the dependence of the recorded mark length on the film thickness, we have simulated the recording process for the medium thickness of 20 $\mathrm{nm}$. Figure 4 shows recorded mark length vs designed mark length simulated for the formation of an isolated mark in the $\mathrm{Tb}_{x}\left(\mathrm{Fe}_{78} \mathrm{Co}_{22}\right)_{100-x}$ media with the thickness of $20 \mathrm{~nm}$. The laser irradiation power is set to $2.0 \mathrm{~mW}$ so that the maximum temperature of the medium becomes almost the same as that for $h=40 \mathrm{~nm}$. Just as the case of $h=40 \mathrm{~nm}$, the recorded mark length decreased with decreasing the designed mark length, and the smallest mark length was obtained for $x=21.7$. In the case of $x=19.4$, the recorded mark length was found to become $96 \mathrm{~nm}$ for the designed mark of $50 \mathrm{~nm}$, which is smaller than that for $h=40 \mathrm{~nm}$. This is due to the decrease of the stray field acting on the domain wall.

\section{References}

[1] N. Takagi, K. Mitani, H. Awano, K. Shimazaki, and N. Ohta: J. Magn. Soc. Jpn., 23 Suppl. S1 (1999) 161.

[2] T. Shiratori, E. Fujii, Y. Miyaoka, and Y. Hozumi: J. Magn. Soc. Jpn., 22 Suppl. S2 (1998) 47.

[3] K. Brugger: J. Appl. Phys., 43 (1972) 577.

[4] B. G. Huth: IBM J. Res. Develop., 18 (1974) 100.

[5] F. Tanaka, S. Tanaka, and N. Imamura: Jpn. J. Appl. Phys., 26 (1987) 231.

[6] N. Takagi, P. Fischer and S. Tsunashima: J. Magn. Soc. Jpn.,25 (2001) 1370.

Received May 7, 2002 ; Accepted June 14, 2002. 